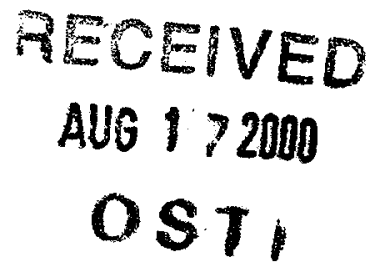

Solid State Communications $114(2000) 63-68$

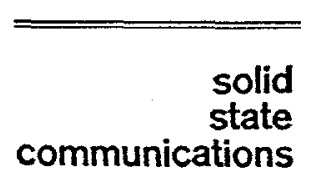

www.elsevier.com/locate/ssc

\title{
Photoluminescence detected enhancement of the electron-hole exchange interaction in a quantum well
}

\author{
F.M. Munteanu ${ }^{\mathrm{ab}, *}$, Y. Kim ${ }^{\mathrm{a}}$, C.H. Perry ${ }^{\mathrm{b}}$, D.G. Rickel ${ }^{\mathrm{a}}$, J.A. Simmons ${ }^{\mathrm{c}}$, J.L. Reno ${ }^{\mathrm{c}}$ \\ ${ }^{2}$ National High Magnetic Field Laboratory, Los Alamos National Laboratory, MS E-536 Los Alamos, NM 87545, USA \\ ${ }^{b}$ Department of Physics, Northeastem University, Boston, MA, USA \\ 'O2115 Sandia National Laboratory, Albuquerque, NM 87185, USA
}

Received 29 November 1999; accepted 23 December 1999 by A.H. MacDonald

\begin{abstract}
We report the results of polarized magneto-photoluminescence (MPL) measurements on a high mobility modulation-doped $\mathrm{GaAs} / \mathrm{AlGaAs}$ single quantum well. The magnetic field was varied between 0 and $60 \mathrm{~T}$. The appearance of a doublet structure in the heavy-hole magneto-exciton in the high field regime was interpreted as an indication for enhanced electron-hole exchange interaction induced by the confinement. The effective $g$-factor of the electron determined from the Zeeman splitting was 3.7 times higher than the bulk value. (C) 2000 Published by Elsevier Science Ltd. All rights reserved.
\end{abstract}

Keywords: A. Quantum wells; A. Semiconductors

The formation and the evolution of the neutral and charged magneto-excitons in GaAs/AlGaAs quantum wells (QWs) and single heterojunctions (SHJs) have been extensively investigated. Since the heavy-hole $(\mathrm{HH})$ and the light-hole (LH) subbands are energetically split, two types of Wannier excitons can be distinguished: the heavy-hole exciton (HHE) and the light-hole exciton (LHE). The Zeeman splitting of the $\mathrm{HH}$ excitons confined in QWs was successfully measured by Snelling et al. [1]. On the contrary, the enhancement of the electron-hole exchange interaction (EHEXI) [2] induced by the 2D confinement has been less investigated. Bauer et al. [3] reported the first evidence for a doublet character of the $\mathrm{HH}$ exciton : recombination, caused by a width-dependent localization enhanced EHEXI in a type I GaAs/AIGaAs QW. They measured the energy of the splitting for different QWs in zero magnetic field as a function of well width and temperature. The lower energy peak of the doublet was shown to come from a dipole forbidden transition as a result of thermalization effects. Similar conclusions were reached by Potemski et al. [4] using a new spectroscopic method to

* Corresponding author. National High Magnetic Field Laboratory, Los Alamos National Laboratory, MS E-536 Los Alamos, NM 87545, USA. Tel.: + 1-505-665-4330; fax: + 1-505-665-4311.

E-mail address: munteanu@lan1.gov (F.M. Munteanu). identify the electronic spin orientation of excitons in a magnetic field [5]. In GaAs/AlGaAs type II multiple QWs, the exchange splitting was investigated by van Kestern et al. [6] using optically detected magnetic resonance (ODMR) experiments. Theoretical investigations $[7,8]$, on the other hand, showed that the observed splittings are too large to be explained by exchange effects. For example, Andreani and Bassani [7] showed that the splitting for a type I QW at zero field should be an order of magnitude smaller than those found experimentally $[3,4]$.

Here we report a series of circularly polarized magnetophotoluminescence (MPL) measurements on a high-quality GaAs/AlGaAs QW with a well of $200 \AA$. The experiments were performed in magnetic fields up to $60 \mathrm{~T}$ and at temperatures between 370 and $1.5 \mathrm{~K}$. The appearance of a high-energy peak in the right circular polarization ( $R C P)$, in addition to the observed HHE and LHE peaks, is considered to be a result of localization enhanced EHEXI. This peak could only be resolved for magnetic fields higher than $9 \mathrm{~T}$ due to the small separation between it and the HHE peak at low magnetic fields. The electron effective $g$-factor was determined to be 3.7 times higher than its bulk value as a result of the EHEXI enhancement. We also observed the formation, around the filling factor $\nu \cong 2$, of an extra peak (H) with energy between the energy of the HHE peak and the one due to the enhanced EHEXI. We followed this peak 


\section{DISCLAIMER}

This report was prepared as an account of work sponsored by an agency of the United States Government. Neither the United States Government nor any agency thereof, nor any of their employees, make any warranty, express or implied, or assumes any legal liability or responsibility for the accuracy, completeness, or usefulness of any information, apparatus, product, or process disclosed, or represents that its use would not infringe privately owned rights. Reference herein to any specific commercial product, process, or service by trade name, trademark, manufacturer, or otherwise does not necessarily constitute or imply its endorsement, recommendation, or favoring by the United States Government or any agency thereof. The views and opinions of authors expressed herein do not necessarily state or reflect those of the United States Government or any agency thereof. 


\section{DISCLAIMER}

Portions of this document may be illegible in electronic image products. Images are produced from the best available original document. 


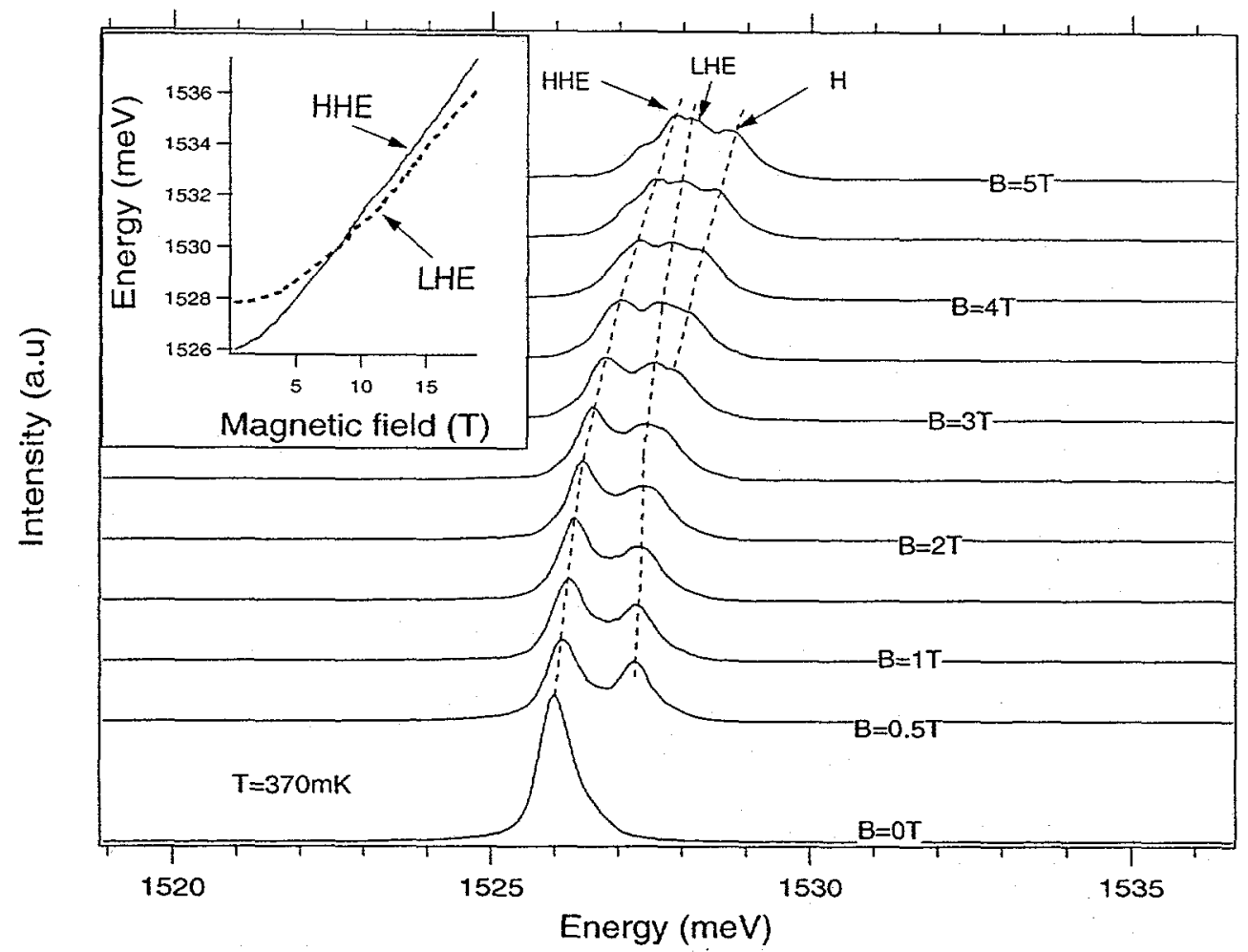

Fig. 1. Unpolarized MPL spectra between 0 and $5 \mathrm{~T}$ at a temperature of $370 \mathrm{mK}$. The light-hole (LHE) and heavy-hole (HHE) exciton states are well resolved at $0.5 \mathrm{~T}$. At a field of about $3 \mathrm{~T}$, a new peak labeled $\mathrm{H}$ appears. The inset shows the diamagnetic energy shift of the LHE and HHE as a function of magnetic field. Beyond $20 \mathrm{~T}$ the LHE peak could no longer be resolved.

all the way up to $60 \mathrm{~T}$ in both RCP and left circular polarizations (LCP). Its behavior with magnetic field and temperature has certain similarities with the some previously reported anomalies in the integer (IQHE) [9] and fractional quantum Hall regime (FQHE) [10,11]. However, up to this point we are unable to offer an unambiguous interpretation of this peak.

The sample that we used in the experiment was a $200 \AA$ $\mathrm{GaAs} / \mathrm{Al}_{0.55} \mathrm{Ga}_{0.45} \mathrm{As}$ single $\mathrm{QW}$ remotely doped. The dark electron density was $1.2 \times 10^{11} \mathrm{~cm}^{-2}$ and the mobility higher than $3 \times 10^{6} \mathrm{~cm}^{2} \mathrm{~V}^{-1} \mathrm{~s}^{-1}$. With constant laser illumination (at $632.8 \mathrm{~nm}$ ) during the measurements, the $2 \mathrm{DEG}$ density increased to $1.58 \times 10^{11} \mathrm{~cm}^{-2}$. Using a quasicontinuous magnet, the field was varied from 0 to $60 \mathrm{~T}$, while the temperature was changed from $1.5 \mathrm{~K}$ to $370 \mathrm{mK}$. Details of the experiment have been described elsewhere [12].

Fig. 1 shows the unpolarized spectra taken at a temperature $T=370 \mathrm{mK}$. Transport measurements under equivalent illumination were used to determine the actual $2 \mathrm{D}$ carrier concentration $n=1.58 \times 10^{11} \mathrm{~cm}^{-2}$. This is slightly higher than the estimated [13] density required $(n=1.0 \times$ $10^{11} \mathrm{~cm}^{-2}$ ) for the unbinding of the excitons at $B=0 \mathrm{~T}$. For the spectra shown in Fig. 1 we find that the peak at $B=0 \mathrm{~T}$ cannot be fitted using a simple Gaussian, due to the broadening on the higher energy side. Following Damen et al. $[14,15]$ we interpret this zero field signal as a mixture between the HHE and the band-to-band recombination which accounts for the high-energy tail. When the magnetic field is turned on, the excitonic character is completely recovered [13]. At $B=0.5 \mathrm{~T}$, a second peak at high energy immediately forms. This peak is due to the LH excitonic states, as a result of the applied magnetic field and was observed in both RCP and LCP. The difference in energy between the LHE and HHE states at very low magnetic field, of about $1.9 \mathrm{meV}$, is comparable with those found in other studies of QWs of about the same size $[16,17]$. The inset in Fig. 1 shows the evolution of the $\mathrm{HH}$ and LH excitons with the magnetic field up to $20 \mathrm{~T}$. Beyond this field the $1 \mathrm{~s}-\mathrm{LHE}$ peak could not be resolved anymore. It can be seen that the two states cross at a field of about $B=8.5 \mathrm{~T}$ as a result of a lighter in-plane mass of the $\mathrm{HH}\left(\cong 0.04 \mathrm{~m}_{0}\right)$ compared with the in-plane mass of the $L H\left(\cong 0.051 \mathrm{~m}_{0}\right.$ ). The ratio of the $E$ vs. $B$ slopes for these two states (in the linear region) gives the ratio of the inverse reduced masses of the excitons. Using the values above for the in-plane HH and LH masses and $0.067 \mathrm{~m}_{0}$ for the electron mass, we find the ratio of the reduced masses $\mu_{\mathrm{LHE}} / \mu_{\mathrm{HHE}}$ to be $\cong 1.16$, in good agreement with the value obtained from the ratio of the slopes.

Due to higher in-plane masses, the LHE is more tightly 

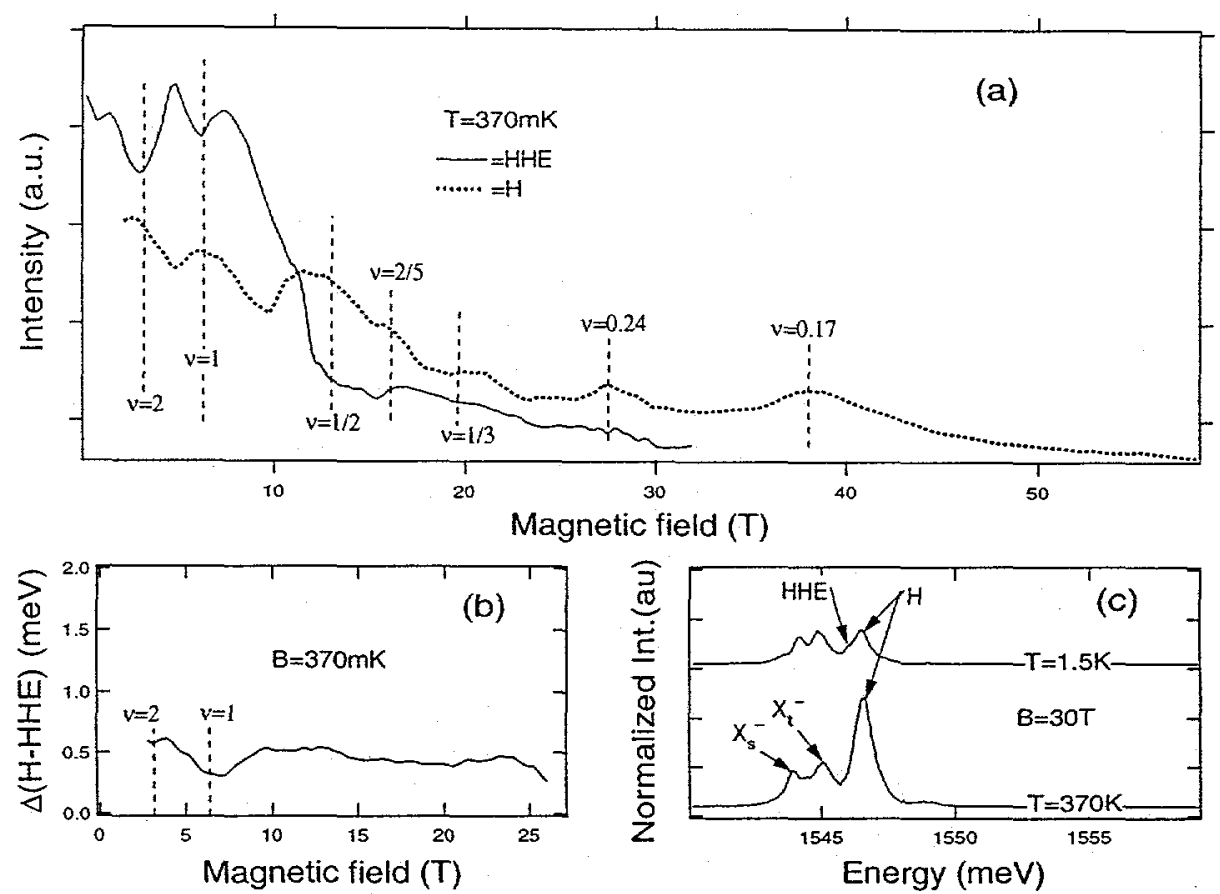

Fig. 2. (a) The evolution of the intensities of the $H$ and HHE peaks with magnetic field. (b) The difference between the energies of the $H$ and HHE peaks. (c) The $B=30 \mathrm{~T}$ spectra at two temperatures $1.5 \mathrm{~K}$ and $370 \mathrm{mK}$.

bound than the HHE. For this reason, the diamagnetic shift in the case of the LHE should be smaller than in the case of the HHE. The energy shift for ls state was shown to be $[18,19]: \Delta E=k B^{2} / \mu^{3}$ (here $\mu$ is the reduced mass of the exciton and $k$ is a constant determined by the exciton state [20]. The diamagnetic shifts that we obtained by fitting the data in the low field regime are: $\Delta E_{\mathrm{LHE}} / B^{2} \sim$ $0.04 \mathrm{meV} \mathrm{T}^{-2}, \Delta E_{\mathrm{HHE}} / B^{2} \sim 0.01 \mathrm{meV} \mathrm{T}^{-2}$.

At a field of about $3 \mathrm{~T}(\nu \cong 2)$ a new high-energy peak, labeled H, appears in the spectrum. In Fig. 2(a), the evolution of the intensities of the HHE and $H$ peaks is shown. The HHE peak displays distinctive minima at filling factors $\nu=1,2$ and 6 which can be related [11] to the suppression of screening and a concomitant increase in the excitonic binding energy. The intensity of the $\mathrm{H}$ peak shows the opposite behavior, with maxima at the filling factors $\nu=2,1$, 0.24 and 0.17 . Less pronounced intensity maxima appear at $\nu=1 / 2,1 / 5$ and $1 / 3$. The difference in energy between $\mathrm{H}$ and $\mathrm{HHE}$ peaks as a function of magnetic field is indicated in Fig. 2(b). This difference is almost constant $(0.5 \mathrm{meV})$, but with a weak minimum at $\nu=2$ and a strong one at $\nu=$ 1. In Fig. 2(c) we show the unpolarized spectra at two temperatures $(370 \mathrm{mK}$ and $1.5 \mathrm{~K})$ normalized with respect the $B=0 \mathrm{~T}$ spectrum. One surprising result is that the intensity of the $\mathrm{H}$ peak is strongly enhanced as the temperature is lowered from $1.5 \mathrm{~K}$ to $370 \mathrm{mK}$. This behavior rules out the possibility that the $\mathrm{H}$ peak is created by fluctuations in the population of the number of the electrons and holes. Above
$30 \mathrm{~T}$, the HHE peak cannot be distinguished due to the high intensity of the $\mathrm{H}$ transition.

Up to this point, we are unable to find an explanation for the appearance of this peak. Its behavior does not strongly depend on polarization, as is the case for the HHE peak whose intensity is reduced in the RCP spectra compared to the LCP spectra. Because the sample was grown with no internuptions, we have ruled out that its formation could be related to imperfections in the size of the QW $[21,22]$. Deveaud et al. [23] showed that a single monolayer disorder can be observed in the PL spectra of GaAs/AlGaAs interfaces. They found that the smallest splitting between the peaks was $4.8 \mathrm{meV}$ in the case of the signal coming from adjacent regions differing in their widths by $a / 2$ (where $a$ is the GaAs lattice constant). This value is about one order of magnitude larger than the splitting that we observe between the $\mathrm{H}$ and HHE peaks. We also have to rule out the possibility of it being created as a result of a field induced HHE spin-splitting. The energy separation between the peaks in our case is almost constant over the entire range of magnetic field considered (see Fig. 2(b)).

Fig. 3(a) shows some spectra taken in the range 8-11 T at a temperature of about $370 \mathrm{mK}$. The most important observation is the appearance in the RCP spectra of a supplementary peak, labeled S. It has an energy higher than the energy of the H peak but is absent in the LCP spectra as illustrated in Fig. 3(b). This figure shows the LCP and RCP spectra taken at $B=10 \mathrm{~T}$ and at the same temperature $370 \mathrm{mK}$. The 

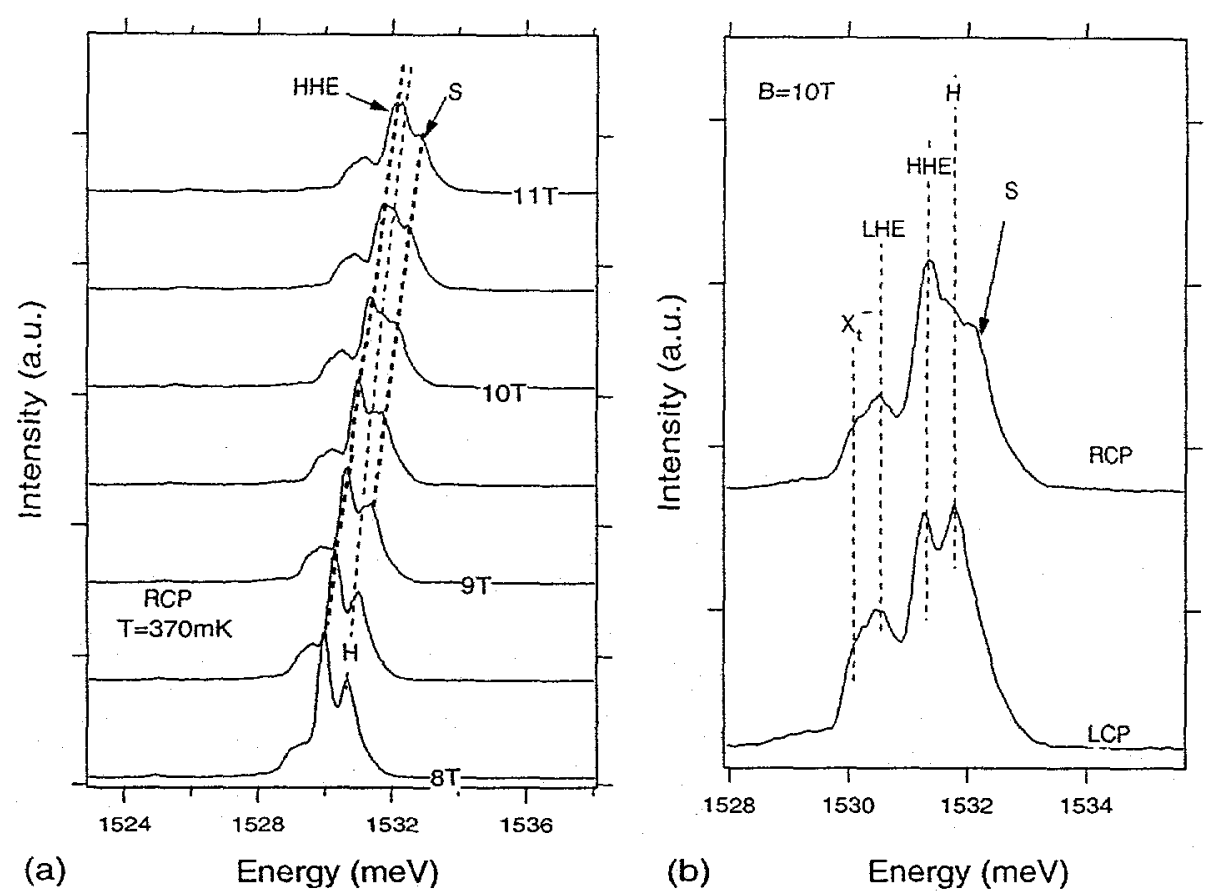

Fig. 3. (a) The RCP spectra show the appearance of the $\mathrm{S}$ peak at $B \cong 9 \mathrm{~T}$. (b) The RCP and LCP spectra at $B=10 \mathrm{~T}$. The $\mathrm{S}$ peak is absent in the LCP spectrum.

peaks, in order from the lowest to the highest energy, are due to the triplet state of the negatively charged magneto-exciton $\left(X_{t}^{-}\right)$, the LHE, the HHE and the H peak, respectively. We interpret this new peak $S$ as evidence of the enhancement of the EHEXI.

Chen et al. [2] showed that the short range of the EHEXI in a QW is considerably enhanced as a result of the confinement, compared with its value in the bulk material. For this reason, the fine structure of the HHE at $B=0 \mathrm{~T}$ consists of two transitions. The higher energy one is dipole allowed and the lower energy one (with the energy equal to the energy of the HHE in the absence of the EHEXI) dipole forbidden. Labeling the excitonic states $\left|m_{\mathrm{h}}, m_{\mathrm{e}}\right\rangle$, the dipole allowed transitions will be [2]: $| \pm 3 / 2, \mp 1 / 2\rangle$ and $| \pm 1 / 2, \pm 1 / 2\rangle$ for the HHE and LHE, respectively. The $|+3 / 2,-1 / 2\rangle$ and $|+1 / 2,+1 / 2\rangle$ states should be RCP polarized, while the $|-3 / 2,+1 / 2\rangle$ and $|-1 / 2,-1 / 2\rangle$ are LCP polarized. The dipole forbidden transition will be given by $| \pm 3 / 2, \pm 1 / 2\rangle$. The appearance of this peak in the PL signal [3] was related to thermalization effects. The selection rules show that for dipole forbidden states to decay, an electronor hole-spin flip is required [6]. Bauer et al. [3] observed that the energy difference between the dipole allowed peak and the dipole forbidden peak (whose energy is the same as the energy of the HHE calculated without EHEXI) is about $0.6 \mathrm{meV}$, for a $\mathrm{QW}$ with the well width of $150 \AA$ at zero magnetic field. These results strongly disagree with the theoretical work presented by Andreani and Bassani [7].
The latter calculated the factor with which the short range exchange interaction is enhanced in QWs over the bulk value as a function of $\mathrm{QW}$ width. Their results, using a value of the bulk exchange-splitting measured earlier $[24,25]$, showed that the energy splitting should be at least ten times smaller than the experimentally observed value. In the case of a $200 \AA \mathrm{QW}$ for example, at zero magnetic field, this splitting should be about $0.04 \mathrm{meV}$.

In Fig. 4(a) we show the evolution of the energies of the HHE and $S$ peaks in the RCP polarization as a function of the magnetic field. Around $B=25 \mathrm{~T}$, the intensity of the $S$ peak became very small as a result of depopulation of the $-1 / 2$ electronic level. At $30 \mathrm{~T}$, the HHE peak is almost completely dominated by the $H$ transition and can no longer be resolved. The peak separation between these two peaks increases almost linearly with the field. We should point out the fact that when they are extrapolated to zero magnetic field, the energy difference between the two peaks is close to zero, as calculated [7]. Due to this small separation energy, we were unable to resolve the formation of the $S$ peak below $B=9 \mathrm{~T}$. In Fig. 4(b), we fitted the energy difference between the $S$ and HHE peaks with a Zeeman energy given by $E_{\mathrm{z}}=g m_{\mathrm{B}} B$. The $g$-factor that we obtained is 3.7 times higher than the bulk absolute value of the $g$-factor for the electron $g_{e}=-0.44$ ). According to Chen et al. [2], the appearance of the HHE peak is due to the formation of the $|+3 / 2,+1 / 2\rangle$ dipole forbidden exciton. We ignore the contribution from the $|-3 / 2,-1 / 2\rangle$ dipole forbidden 

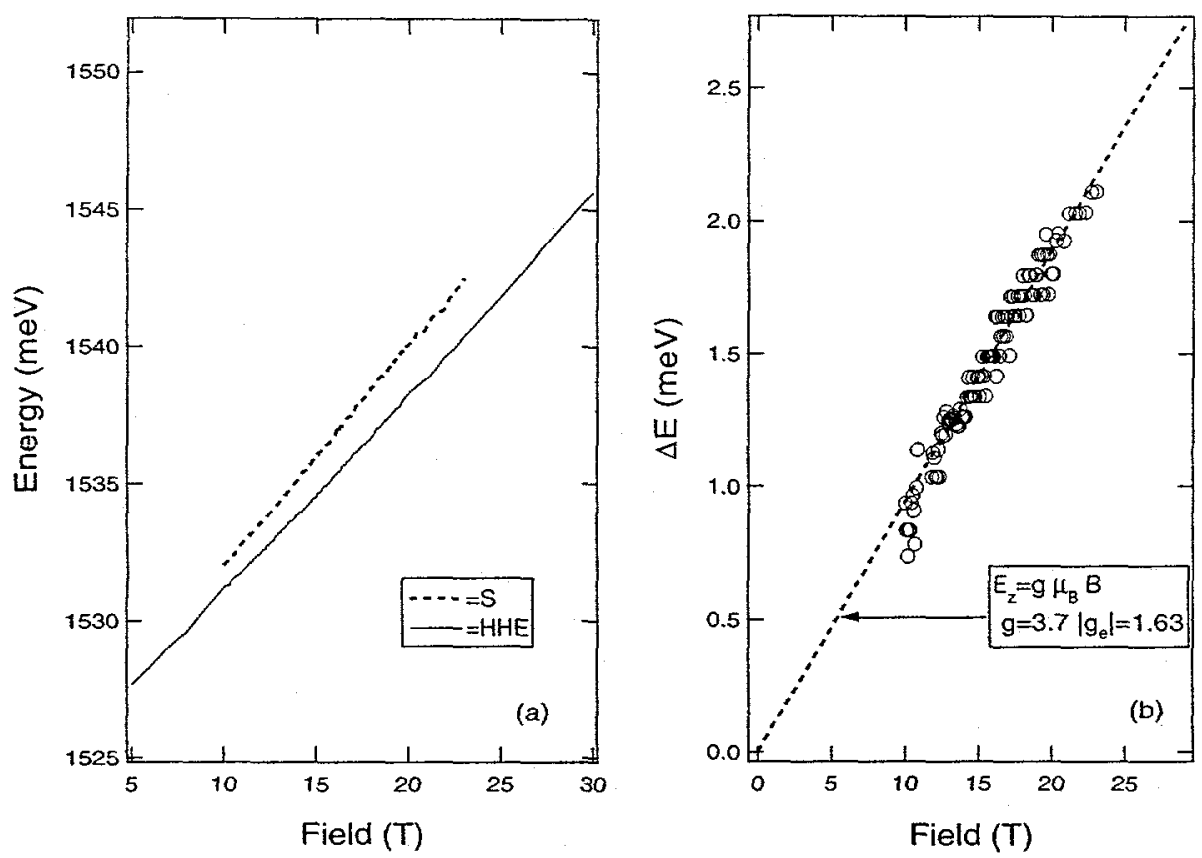

Fig. 4. (a) The evolution of the energies of the HHE (shown in the linear region only) and the S peaks with magnetic field. The H and LHE lines are omitted for clarity; (b) the energy difference between the $S$ and HHE peaks. The continuous line is a fit to the experimental data.

exciton for two reasons: (a) at $\nu=2(B=3.25 \mathrm{~T})$, the $-1 / 2$ electron level begins to depopulate and also, most of the photo-created holes will be located on the $+3 / 2$ spinlevel; (b) in the case when the $|-3 / 2,-1 / 2\rangle$ exciton was present, the Zeeman splitting of the HHE peak should be much larger than that observed. The formation of the HHE peak in the MPL takes place as a result of an electron or hole spin-flip [6] in the initial $|+3 / 2,+1 / 2\rangle$ excitonic state. An electron spin-flip will give an RCP polarized signal, while a hole spin-flip will give an LCP signal. As the LCP signal is stronger than the RCP signal, we conclude that a hole spinflip is energetically more favorable at large fields. This can be as a result of the enhancement of the electron $g$-factor due to the EHEXI, as shown by Nicholas et al. [26], as well as by Lefebvre et al. [27]. From our data we conclude that the electren $g$-factor is enhanced 3.7 times over its bulk value. On the other hand, there is no predicted enhancement in the HH $g$-factor, which can explain why we do not see any signal in the LCP polarization from the initial $|-3 / 2,+1 / 2\rangle$ excitonic state. The Zeeman energy separation between it and the HHE peak produced by the $\mid+3 / 2,+1 / 2$ ) initial excitonic state is much smaller than the one between the HHE peak and the $|+3 / 2,-1 / 2\rangle$ initial excitonic state.

- In conclusion, MPL measurements were performed on a high-quality GaAs/AlGaAs QW. The results show the appearance of a RCP polarized high-energy peak (S), which we interpret as evidence for the enhancement of the EHEXI in agreement with previous theoretical work $[2,7]$. From our data, we estimate that the effective $g$-factor of the electron is 3.7 times the bulk value. Of equal interest is the appearance of an additional peak $(\mathrm{H})$ around $\nu=2$, with an energy between that of the HHE and S peaks. Its intensity is relatively insensitive to polarization and it becomes the dominant peak beyond $30 \mathrm{~T}(\nu \cong 1 / 5)$. It shows intensity oscillations in the IQHE and FQHE regimes that are correlated with the intensity oscillations of the HHE peak, while the energy separation between these two lines remains almost constant. Up to now, we could find no satisfactory explanation for the appearance of this peak.

\section{Acknowledgements}

The authors gratefully acknowledge the engineers and technicians at NHMFL-LANL in the operation of the $60 \mathrm{~T}$ QC magnet. Work at NHMFL-LANL is supported by NSF Cooperative Agreement DMR-9527035, the Department of Energy and the State of Florida. Work at Sandia National Laboratory is supported by the Department of Energy.

\section{References}

[1] M.J. Snelling, E. Blackwood, C.J. McDonagh, R.T. Harley, C.T.B. Foxon, Phys. Rev. B 45 (1992) 3922.

[2] Y. Chen, B. Gil, P. Lefebvre, H. Mathieu, Phys. Rev. B 37 (1988) 6429.

[3] R. Bauer, D. Bimberg, J. Christed, D. Oertel, D. Mars, 
J.N. Miller, T. Fukunaga, H. Nakashima, in: O. Engstrom (Ed.), Proceedings of the 18th International Conference on Semiconductor Physics, Stockholm, 1986, World-Scientific, Singapore, 1987, p. 525.

[4] M. Potemski, J.C. Maan, A. Fasolino, K. Ploog, G. Weimann, Surf. Sci. 229 (1990) 151-154.

[5] M. Potemski, J.C. Maan, A. Fasolino, K. Ploog, G. Weimann, Phys. Rev. Lett. 63 (1989) 2409.

[6] H.V. van Kestern, E.C. Cosman, F.J.A.M. Greidanus, P. Dawson, K.J. Moore, C.T. Foxon, Phys. Rev. Lett. 61 (1988) 129.

[7] L.C. Andreani, F. Bassani, Phys. Rev. B 41 (1990) 7536

[8] B.R. Salmassi, G.E.W. Bauer, Phys. Rev. B 39 (1989) 1970.

[9] F.M. Munteanu, Y. Kim, C.H. Perry, D.G. Rickel, J.A. Simmons, J.L. Reno, Solid State Commun. 111 (1999) 605.

[10] D. Heiman, B.B. Goldberg, A. Pinczuk, C.W. Tu, A.C. Gossard, J.H. English, Phys. Rev. Lett. 61 (1988) 605.

[11] A.J. Turberfield, S.R. Haynes, P.A. Wright, R.A. Ford, R.G. Clark, J.F. Ryan, Phys. Rev. Lett. 65 (1990) 637

[12] C.H. Perry, Y. Kim, D.G. Rickel, Physica B 246-247 (1998) 182.

[13] G.E.W. Baver, Phys. Rev. B 45 (1992) 9153.

[14] T.C. Damen, J. Shah, D.Y. Oberli, D.S. Chemla, J.E. Cunningham, J.M. Kuo, J. Lumin. 45 (1990) 181-185.

[15] T.C. Damen, J. Shah, D.Y. Oberli, D.S. Chemla J.E. Cunningham, J.M. Kuo, Phys. Rev. B 42 (1990) 7434.
[16] A.J. Stields, J.L. Osborne, D.M. Whittaker, M.Y. Simmons, M. Pepper, D.A. Ritchie, Phys. Rev. B 55 (1997) 1318.

[17] W.T. Masselink, P.J. Pearah, J. Klem, C.K. Peng, H. Morkoc, Phys. Rev. B 32 (1985) 8027.

[18] S. Tarucha, H. Okamoto, Y. Iwasa, N. Miura, Solid State Commun. 52 (1984) 815-819.

[19] D.C. Rogers, J. Singleton, R.J. Nicholas, C.T. Foxon, K. Woodbridge, Phys. Rev. B 34 (1986) 4002.

[20] D.C. Reynolds, T.C. Collins, Excitons, their Properties and Uses, Academic Press, New York, 1981, p. 31 (chap. 2).

[21] C.W. Tu, R.C. Miller, B.A. Wilson, P.M. Petroff, T.D. Harris, R.F. Kopf, S.K. Sputz, M.G. Lanont, J. Cryst.Growth 81 (1987) 159-163.

[22] R.C. Miller, C.W. Tu, S.K. Sputz, R.F. Kopf, Appl. Phys. Lett. 49 (1986) 1245

[23] B. Deveaud, J:Y. Emery, A. Chomette, B. Lambert, M. Baudet, Appl. Phys. Lett. 45 (10) (1984) 1078.

[24] W. Ekardt, K. Losch, D. Bimberg, Phys. Rev. B 20 (1979) 3303.

[25] R. Sooryakumar, P.E. Simmonds, Phys. Rev. B 27 (1983) 4978.

[26] R.J. Nicholas, R.J. Haug, K.V. Klitzing, G. Weiman, Phys. Rev. B 37 (1988) 1294.

[27] P. Lefebvre, B. Gil, J.P. Lascaray, H. Mathieu, D. Bimberg, T. Fukunaga, H. Nakashima, Phys. Rev. B 37 (1988) 4171. 